\section{Case Reports in Ophthalmology}

Case Rep Ophthalmol 2020;11:249-255

DOI: $10.1159 / 000508067$

Published online: June 29, 2020
(C) 2020 The Author(s)

Published by S. Karger AG, Basel www.karger.com/cop

\title{
Removal of a Baerveldt Glaucoma Implant and Fibrous Adhesion for Refractory Mechanical Strabismus
}

\author{
Mai Ueda Morino Tadamichi Akagi Manabu Miyata \\ Akitaka Tsujikawa \\ Department of Ophthalmology and Visual Sciences, Kyoto University Graduate School of \\ Medicine, Kyoto, Japan
}

\section{Keywords}

Glaucoma - Glaucoma implant surgery - Mechanical strabismus - Eye movement disorder .

Implant removal

\begin{abstract}
Although strabismus is a well-known complication of glaucoma implant surgery, its surgical treatment is still challenging. We present a case with refractory strabismus after Baerveldt glaucoma implant (BGI) surgery, which was not sufficiently improved by strabismus surgery, but by removal of the BGI and fibrous adhesion. The patient was a 35-year-old woman who had multiple surgeries for secondary glaucoma. She had severe limitations of the movement of her right eye and binocular diplopia in all gaze positions after BGI surgery. Although she underwent two strabismus surgeries, the strabismus was not resolved. The surgery was performed by two specialists in glaucoma and strabismus. The BGI plate and the fibrous capsule were carefully removed. The fibrous tissue involved the muscle bellies of the inferior and particularly lateral rectus (LR) muscles and induced tight and wide adhesion between the muscle bellies and sclera. The adhesion was released after tenotomy at the insertion of the LR muscle, and the LR muscle was additionally resected by $6.0 \mathrm{~mm}$. The ocular position and movement dramatically improved; however, intraocular pressure (IOP) increased immediately after the surgery. Then, Ahmed glaucoma valve implantation was performed 5 days after BGI removal. The IOP decreased and has been controlled. The improvement of ocular position and movement
\end{abstract}




\section{Case Reports in Ophthalmology}

Case Rep Ophthalmol 2020;11:249-255

Morino et al.: Removal of a BGI and Fibrous Adhesion for Refractory Mechanical Strabismus

remained 9 months postoperatively and she obtained cosmetic satisfaction without diplopia or worsening of visual acuity. Removal of BGI and fibrous adhesion is a potential option for refractory mechanical strabismus following BGI surgery; however, it is important to prepare additional procedures for the subsequent IOP increase in advance.

(C) 2020 The Author(s)

Published by S. Karger AG, Basel

\section{Introduction}

Diplopia with motility disturbance is one of the complications of implanted glaucoma drainage devices (GDDs), such as the Baerveldt glaucoma implant (BGI). In the Tube Versus Trabeculectomy (TVT) study, persistent diplopia was reported in 5\% of patients 1 year after BGI surgery [1]. A recent prospective study showed that diplopia was experienced by $27 \%$ and changes in duction were observed in 35\% of patients 1 year after BGI surgery [2], which suggests that mechanical strabismus is commonly induced after BGI surgery. Strabismus surgery with preservation of the filtering bleb related to a GDD can reportedly be successful in many cases [3]; however, complete removal of the fibrous capsule surrounding the implant and involved adjacent structures might be required in some severe cases [4]. Here, we present a patient with refractory strabismus after BGI surgery, which was not sufficiently improved by strabismus surgery performed on four muscles, but by removal of the BGI and fibrous capsule.

\section{Case Presentation}

A 35-year-old Japanese woman with secondary glaucoma had previous surgeries including a cataract surgery, pars plana vitrectomy, two trabeculotomies, and a trabeculectomy in her right eye. She underwent BGI surgery in the inferotemporal region at 32 years of age due to high intraocular pressure (IOP). She had severe limitations of the movement of her right eye and binocular diplopia in all gaze positions after BGI surgery. She underwent two strabismus surgeries: (1) 7.0-mm medial rectus muscle recession and 3.0-mm superior rectus muscle recession and (2) 4.0-mm lateral rectus (LR) muscle resection and 3.0-mm inferior rectus muscle resection. However, the strabismus was not resolved. She felt extreme cosmetic discomfort and sometimes felt diplopia. Although she visited several hospitals for treatment, she could not obtain satisfaction. Finally, she came to our hospital for treatment. The information received from the previous clinic indicated that the second strabismus surgery was complicated because of excessive adhesions around the BGI plate and resulted in very limited treatment effects.

At the initial visit to our hospital, the patient's best-corrected visual acuity was 20/40 in the right eye and 20/13 in the left eye. The IOPs were $20 \mathrm{~mm}$ Hg with ocular hypotensive drops and $15 \mathrm{~mm} \mathrm{Hg}$ without treatment. The visual field was severely damaged in the right eye, but it was within normal limits in the left eye (Fig. 1). She had remarkably limited right eye movement, especially for abduction and infraduction (Fig. 2a). After obtaining informed consent after explaining the probability of vision loss due to postoperative IOP increase, the surgery was performed by two specialists in glaucoma (T.A.) and strabismus (M.M.). The fibrous capsule surrounding the BGI was carefully removed (Fig. 3a-c) and the BGI plate was removed (Fig. 3d). Because the fibrous tissue involved the muscle bellies of the inferior and particularly LR muscles and induced tight and wide adhesion between the muscle bellies and 


\section{Case Reports in Ophthalmology}

Case Rep Ophthalmol 2020;11:249-255

DOI: $10.1159 / 000508067$

C 2020 The Author(s). Published by S. Karger AG, Basel www.karger.com/cop

Morino et al.: Removal of a BGI and Fibrous Adhesion for Refractory Mechanical Strabismus

sclera, we intraoperatively judged that the LR muscle did not function mechanically. The adhesion was released after tenotomy at the insertion of the LR muscle (Fig. 3e, f) for the purpose of procedure safety, including avoidance of scleral perforation. The LR muscle was additionally resected by $6.0 \mathrm{~mm}$ because postoperative residual esodeviation would inhibit binocularity. The ocular position and movement dramatically improved and the patient felt satisfied. However, IOP increased up to $48 \mathrm{~mm} \mathrm{Hg}$ immediately after the surgery as we expected. Because the IOP was not sufficiently controlled with glaucoma medications, Ahmed glaucoma valve implantation was performed in the inferonasal region 5 days after BGI removal. We selected the inferonasal region for this surgery because of the fibrous scarring caused by the inferotemporal BGI surgery. Additionally, the previous superotemporal trabeculectomy scar adversely affected the superotemporal region. The IOP decreased and has been controlled since (17 mm Hg 9 months postoperatively). The improvement of ocular position and movement remained 9 months postoperatively and she obtained cosmetic satisfaction without diplopia or worsening of visual acuity (Fig. $2 \mathrm{~b}$ ).

\section{Discussion}

The surgical treatment of strabismus following implantation of GDDs is often challenging. In mild cases, conservative treatment such as wearing prism glasses can be an option, but in severe cases, which are rarely observed, surgery is a potential option. The most frequent reason for strabismus was reported to be a stretched muscle over the GDD or posterior fixation effect caused by scarring posterior to the GDD [4, 5]. In cases with fibrous scarring inhibiting extraocular muscle movement, strabismus surgery alone would be less effective, and removal of the fibrous adhesion might be necessary. In more severe cases, removal of the GDD should also be considered.

Strabismus following BGI in the superotemporal quadrant possibly results from exotropia and hypertropia, caused by stretching of the muscle over the GDD [5, 6]. However, our case of inferotemporal BGI implantation showed esotropia and hypertropia. It is unclear why our case did not exhibit exotropia and hypotropia. We suspect that there was an anterior and posterior fixation effect induced by scarring between the muscle belly and the sclera. This may have been a major cause of strabismus in our case. The LR muscle belly was tightly fixed and ocular motility was extremely limited at all gaze positions due to fibrous adhesions. Considering that there were already excessive adhesions at the time of the second strabismus surgery in the other hospital, removal of fibrous adhesions was needed. Furthermore, it is also possible that the previous strabismus surgeries complicated the patient's pathological condition.

Removal of BGI and fibrous adhesion surrounding the LR muscle belly was dramatically effective. We selected the inferonasal region for Ahmed glaucoma valve implantation after removal of the BGI, although other options existed. One such option may involve cutting the wings of the BGI and putting it back into the original location. Alternatively, a smaller BGI or Ahmed glaucoma valve could be placed into the same area. In our case, considering the extreme scarring and the damaged LR, we estimated that the inferonasal region was the preferred location. However, there might be room for further discussion on the potential methods and locations of additional surgeries. Furthermore, there is a need to discuss whether additional strabismus surgery combined with the removal of BGI is necessary. We additionally performed LR resection based on our institutional criteria because residual esodeviation could cause diplopia postoperatively. However, it induced a small overcorrection. An adjustable suture technique might be an option for additional strabismus surgery. 


\section{Case Reports in Ophthalmology}

In conclusion, removal of BGI and fibrous adhesion is a potential option for refractory mechanical strabismus following BGI surgery; however, it is important to prepare additional procedures for the subsequent IOP increase in advance.

\section{Statement of Ethics}

Written informed consent was obtained from the patient for publication of this case report and any accompanying images.

\section{Disclosure Statement}

The authors declare that there is no conflict of interest regarding the publication of this paper.

\section{Funding Sources}

This work was supported by the Japan Society for the Promotion of Science (JSPS) KAKENHI grant No. 16K11267 (T.A.). The funder had no role in the study design, data collection, analysis, the decision to publish, or in the preparation of the manuscript.

\section{Author Contributions}

Mai Ueda Morino contributed to the data acquisition, literature search, and manuscript draft. Tadamichi Akagi performed the surgery and was a major contributor in the conception and design, interpretation of data, and writing the manuscript. Manabu Miyata performed the surgery and was involved in drafting and revising the manuscript. All authors were directly involved in the care of the patient and read and approved the final manuscript.

\section{References}

1 Rauscher FM, Gedde SJ, Schiffman JC, Feuer WJ, Barton K, Lee RK; Tube Versus Trabeculectomy Study Group. Motility disturbances in the tube versus trabeculectomy study during the first year of follow-up. Am J Ophthalmol. 2009 Mar;147(3):458-66.

2 Islamaj E, Jordaan-Kuip CP, Vermeer KA, Lemij HG, de Waard PW. Motility Changes and Diplopia After Baerveldt Glaucoma Drainage Device Implantation or After Trabeculectomy. Transl Vis Sci Technol. 2018 Sep;7(5):7.

3 Osigian CJ, Cavuoto KM, Rossetto JD, Sayed M, Grace S, Chang TC, et al. Strabismus surgery outcomes in eyes with glaucoma drainage devices. J AAPOS. 2017;21(2):103-6.e2.

4 Roizen A, Ela-Dalman N, Velez FG, Coleman AL, Rosenbaum AL. Surgical treatment of strabismus secondary to glaucoma drainage device. Arch Ophthalmol. 2008 Apr;126(4):480-6.

5 Muñoz M, Parrish RK 2nd. Strabismus following implantation of Baerveldt drainage devices. Arch Ophthalmol. 1993 Aug;111(8):1096-9.

6 Smith SL, Starita RJ, Fellman RL, Lynn JR. Early clinical experience with the Baerveldt 350-mm2 glaucoma implant and associated extraocular muscle imbalance. Ophthalmology. 1993 Jun;100(6):914-8. 
Case Reports in Ophthalmology www.karger.com/cop

Morino et al.: Removal of a BGI and Fibrous Adhesion for Refractory Mechanical Strabismus
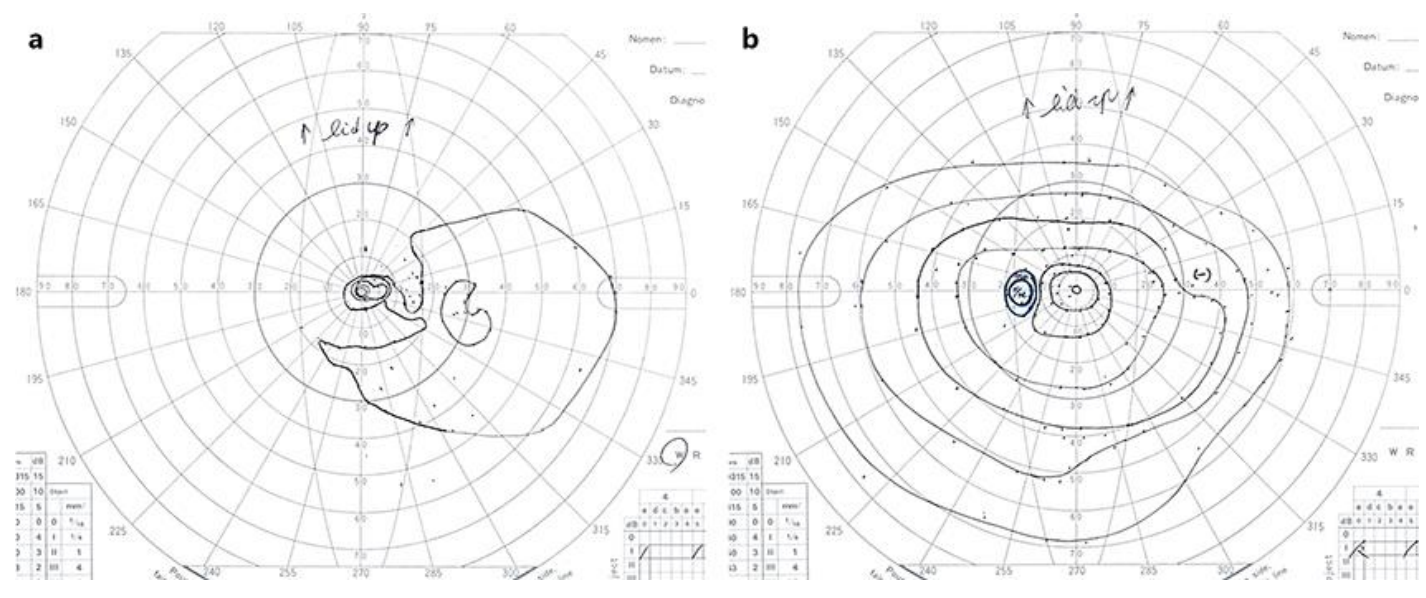

Fig. 1. Goldmann visual field measurements at the patient's initial visit to our hospital. a The visual field of the right eye is severely damaged. $\mathbf{b}$ The visual field of the left eye is within normal limits. 


\section{Case Reports in Ophthalmology}
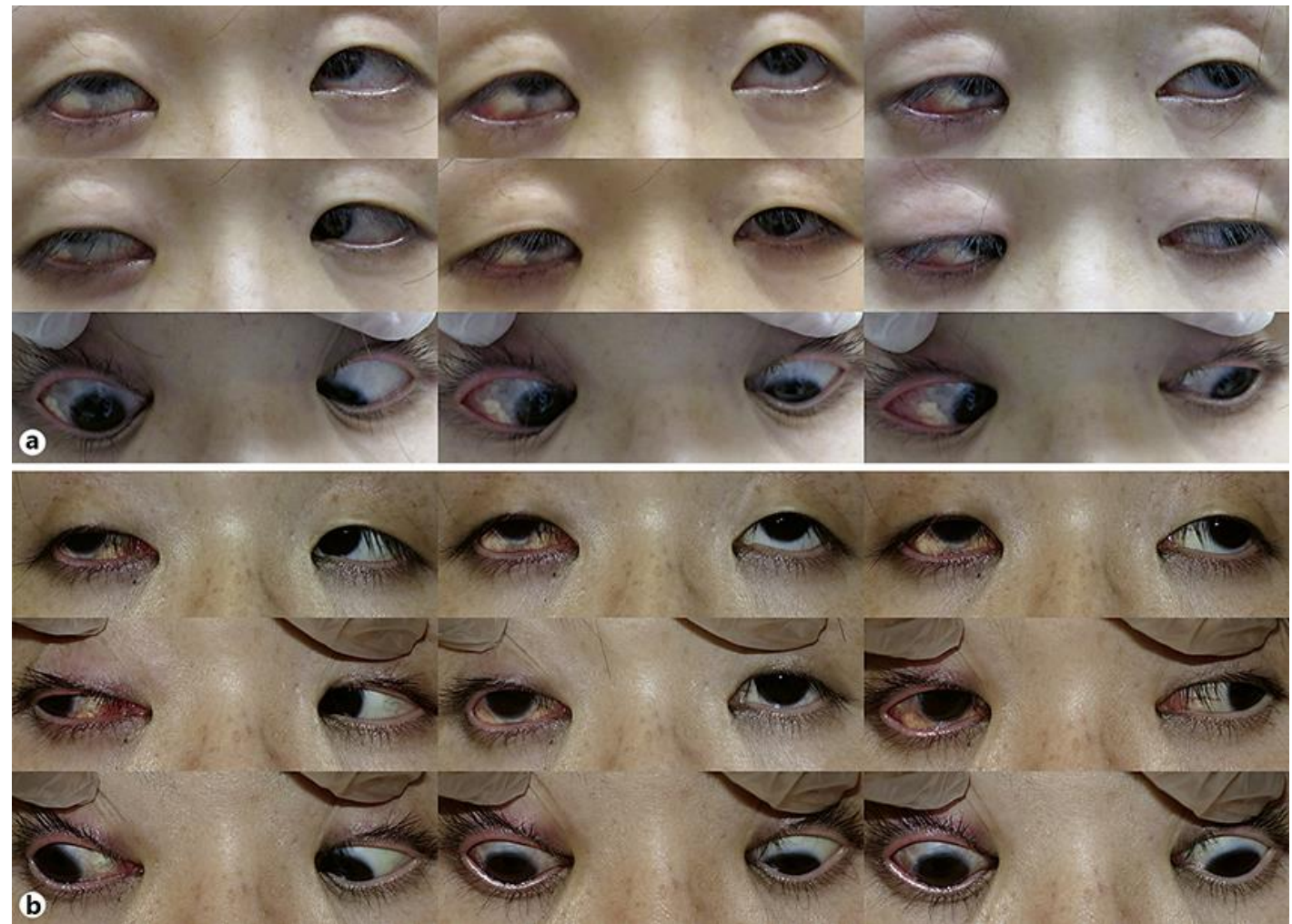

Morino et al.: Removal of a BGI and Fibrous Adhesion for Refractory Mechanical Strabismus

Fig. 2. Ocular alignment in a patient with glaucoma-implant-induced mechanical strabismus. a Before removal of a glaucoma implant and fibrous adhesion in her right eye. Right esodeviation $(14 \Delta)$ and right hyperdeviation $(25 \Delta)$ in the primary gaze position and severe restriction of abduction and infraduction are observed in her right eye. b Postoperative images ( 9 months after surgery). Eye movement is dramatically improved in her right eye. The primary position is right exodeviation $(14 \Delta)$ and right hyperdeviation (4 $\Delta$ ). 


\section{Case Reports in Ophthalmology}
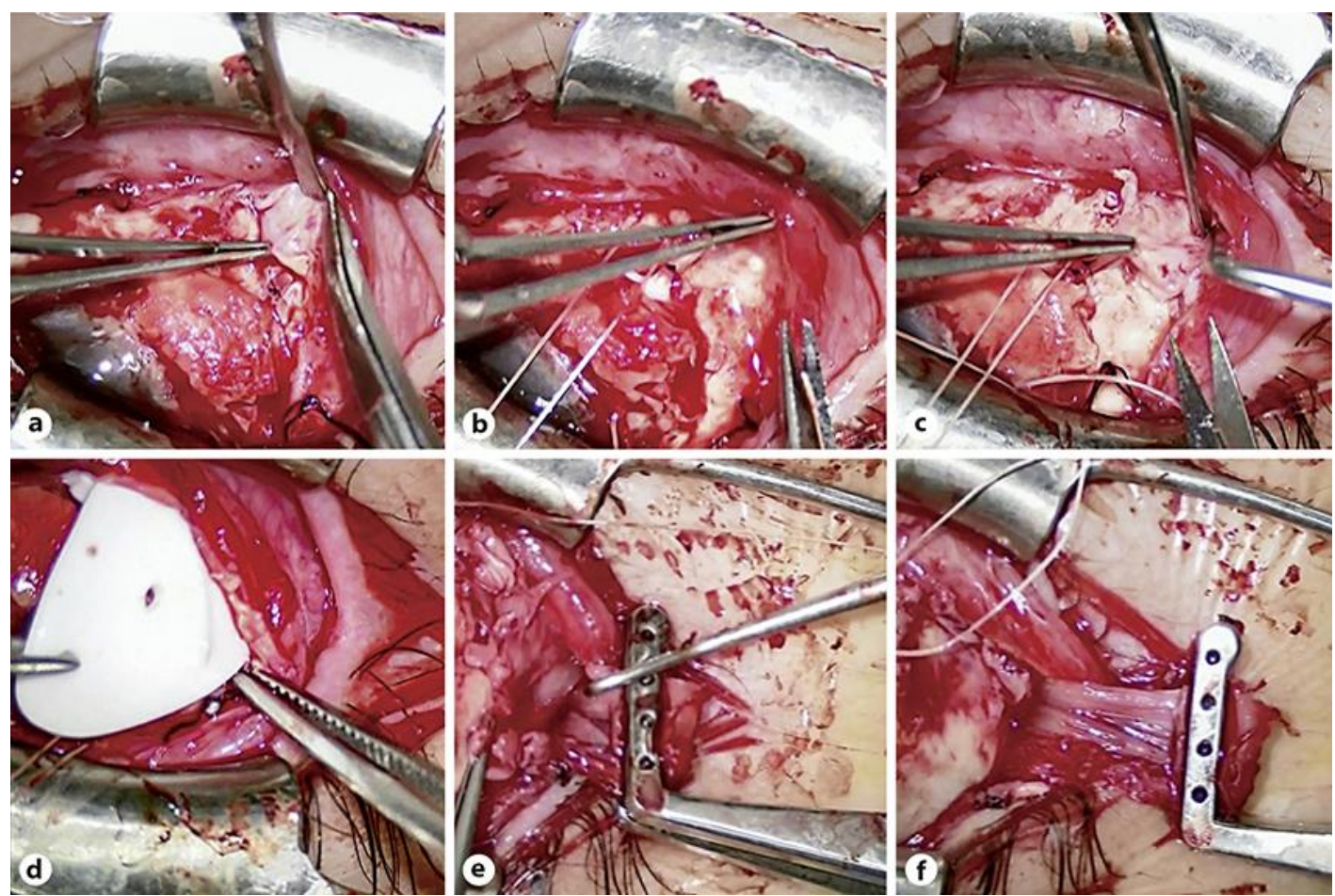

Fig. 3. Intraoperative images. a The fibrous capsule surrounding the Baerveldt glaucoma implant (BGI) is removed. $\mathbf{b}$ A large amount of fluid is gushing out from inside the encapsulation surrounding the BGI after incision. c Fibrous tissue is further removed. $\mathbf{d}$ The BGI plate is removed after dissection of the column structure through the BGI plate. e, $\mathbf{f}$ The adhesion is released after tenotomy at the insertion of the lateral rectus muscle to approximately $12 \mathrm{~mm}$ from the muscle insertion. 